\section{Sarkoidose: Männer erkranken früher}

Arkema EV et al. Sarcoidosis incidence and prevalence: a nationwide register-based assessment in Sweden. Eur Resp J 2016; 48: 1690 - 1699

Die Angaben zur Inzidenz und Prävalenz der Sarkoidose variieren ebenso wie unterschiedliche Geschlechtsund Altersabhängigkeiten. Bisherige Daten stammten überwiegend aus Röntgenregistern. Arkema et al. gingen darüber hinaus und überprüften die epidemiologischen Aspekte mit dem schwedischen Krankenregister. Sie nahmen alle Personen in die landesweite Studie auf, bei denen die Diagnose mindestens 1-mal ambulant oder stationär gestellt wurde.

Am Stichtag 31. Dezember 2013 waren 7692386 Einwohner > 18 Jahre alt. 16 547 Patienten hatten unter dem Stichwort „Sarkoidose“ eine positive Anamnese. Die Prävalenz betrug 215/100000 Personenjahre. Unter der Voraussetzung von $\geq 2$ Treffern pro Patient betrug die Erkrankungshäufigkeit 160/100000 Personenjahre. Zwei Drittel hatten innerhalb von 6 Monaten Medikamente gegen die Sarkoidose erhalten. Dazu gehörten u. a. Kortikosteroide, Antimalariamittel und Immunmodulatoren. Das Durchschnittsalter betrug 56 Jahre. Männer waren mit $56 \%$ gering häufiger betroffen. Die Prävalenz betrug für Frauen 141/100000 und für Männer 179/100000 Personenjahre. Dabei bestanden regionale Unterschiede. Die höchsten Raten ergaben

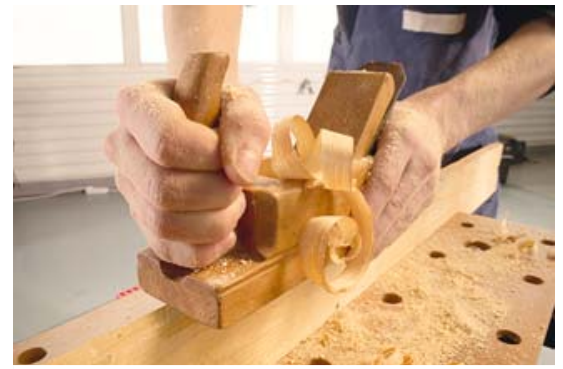

In der schwedischen Studie erkrankten Männer, die in der Landwirtschaft oder Holzindustrie tätig waren, häufiger und früher an Sarkoidose.

sich für die dünn besiedelten nördlichen Landkreise. Im Südosten erkrankten weniger Einwohner an einer Sarkoidose. Die geografische Varianz soll durch die höhere ethnische Diversität in den südlich gelegenen Großstädten bedingt sein. Die stärkere Belastung durch organische Stäube und Holzverbrennung im ländlich geprägten Norden spricht für die Bedeutung von Umwelteinflüssen für die Entstehung der Sarkoidose. Für einen pathophysiologischen Zusammenhang zwischen Sarkoidose und einer Bakteriämie nach Zeckenbissen fanden sich keine Hinweise, denn die Überträger sind eher in den südlichen Regionen verbreitet.

Die Autoren ermittelten die Neuerkrankungsrate für die Jahre 2003 -2012. Die Inzidenz betrug insgesamt 11,5/100000 Personenjahre, wenn 2 Arztkonsultationen wegen einer Sarkoidose erfolgt waren. Die Neuerkrankungsrate lag damit unter früheren Berechnungen. Die Analyse von Röntgenbildern habe auch asymptomatische Zufallsbefunde erfasst, die der Stichwortsuche entgingen.
Jährlich erkrankten durchschnittlich 1079 Personen. Die Inzidenz änderte sich über den Gesamtzeitraum nicht signifikant. Neu Betroffene waren durchschnittlich 50 Jahre alt und 55\% Männer. Verglichen mit den Frauen erkrankten Männer deutlich früher (44,9 Jahre vs. 54,0 Jahre). Der Inzidenz-Peak lag bei den Männern zwischen 30 und 50 Jahren, bei den Frauen erst in der 6 . Lebensdekade. Der im Unterschied zu anderen Studienresultaten späte Krankheitsbeginn wird ebenfalls auf nicht eingeschlossene, klinisch inapparente Fälle zurückgeführt. Der Bildungsstand beeinflusste die Krankheitshäufigkeit nicht.

FAZIT

Die Arbeitsgruppe stellt für die geschlechtsspezifischen Unterschiede u. a. 2 Hypothesen auf: Die unterschiedliche Umweltexposition von Männern und Frauen, z. B. durch die Tätigkeit in der Landwirtschaft oder in der Holzindustrie, erkläre die höhere Erkrankungsrate, den früheren Krankheitsbeginn und den häufigeren Lungenbefall bei Männern. Alternativ sei eine protektive Wirkung durch weibliche Hormone denkbar. Folgestudien sollten geschlechtsspezifische Risikofaktoren ermitteln, so die Autoren.

Dr. med. Susanne Krome, Melle 